\title{
Deformation-Electromagnetic Relations in Lithospheric Activity Manifestations
}

\author{
Igor Larionov ${ }^{1}$, Evgeny Malkin ${ }^{1}$ and Vladimir Uvarov ${ }^{1,1}$ \\ ${ }^{1}$ Institute of Cosmophysical Research and Radio Wave Propagation (IKIR) FEB RAS, 684034, \\ Paratunka, Kamchatksiy kray, Russia
}

\begin{abstract}
It has been shown that dipole radiation of accelerated charges, described by Larmor relation, is the basis of the known mechanicelectromagnetic processes of rock deformation. Comparison of crust deformation acceleration with natural electromagnetic field parameters of ELF-VLF range showed good relation. It manifests in the maxima of occurrence frequency density of synchronous deformation-electromagnetic events on two dimensional histograms. The data of a laser strain-meter and a recorder of natural electromagnetic radiation of ELF-VLF range, recorded in a zone of increased seismic activity (Kamchatka, Karymshina site), were used. The authors made an assumption on the existence of stationary regions of deformation process and mechanic-electromagnetic transformations corresponding to regions with different mechanic properties and rock petrographic composition.
\end{abstract}

Key words: seismoelectromagnetizm, crust deformation-electromagnetic radiation, natural electromagnetic field of ELF-VLF range, seismic activity, rock electromagnetic and mechanic properties, lithosphere electrodynamics

\section{INTRODUCTION}

The phenomenon of electromagnetic manifestation of the Earth crust deformation activity is known quite well and is widely discussed in scientific literature $[1,2]$.

To explain the observed seismo-electromagnetic phenomena, a series of mechanicelectromagnetic mechanisms of mechanic energy transformation into electromagnetic one was suggested: dislocational flows in rock-forming mineral crystals, activation of charge mobile carries, piezoelectric, piezomagnetic, electrokinetic, triboelectric, inductive, inertial, seismomagnetic and other effects $[3,4,5]$.

However, all these effects are various sides of electromagnetic manifestation of matter polarization change under mechanic effect appearing in the form of various effects

${ }^{1}$ Corresponding author: uvarovvnng@yandex.ru 
depending on petrographic situation. Such an approach allows us to use the Larmor relation as the most general description of generated radiation [6]:

$$
d W=\frac{2}{3} \frac{(\ddot{P})^{2}}{c^{3}} d v .
$$

$\vec{P}=Q \cdot \vec{r}$ is the resultant dipole moment of a system,

$Q$ is the charge,

$\boldsymbol{r}$ is the effective base of system dipole.

we see that electric-deformation radiation is the function of acceleration/velocity of deformation and acceleration/velocity of charge generation:

$$
\ddot{\vec{P}}=Q \cdot \ddot{\vec{r}}+2 \dot{Q} \cdot \dot{\vec{r}}+\ddot{Q} \quad \vec{r}
$$

In other words, electromagnetic manifestation of crust deformation processes contains information both on the crust mechanic motion parameters and on the crust electrophysical properties in the areas of deformation activity. It was confirmed in the paper [7] from which we illustrate Fig. 1 demonstrating the correlation of electric field horizontal component amplitude in the ground with shift velocity and acceleration.
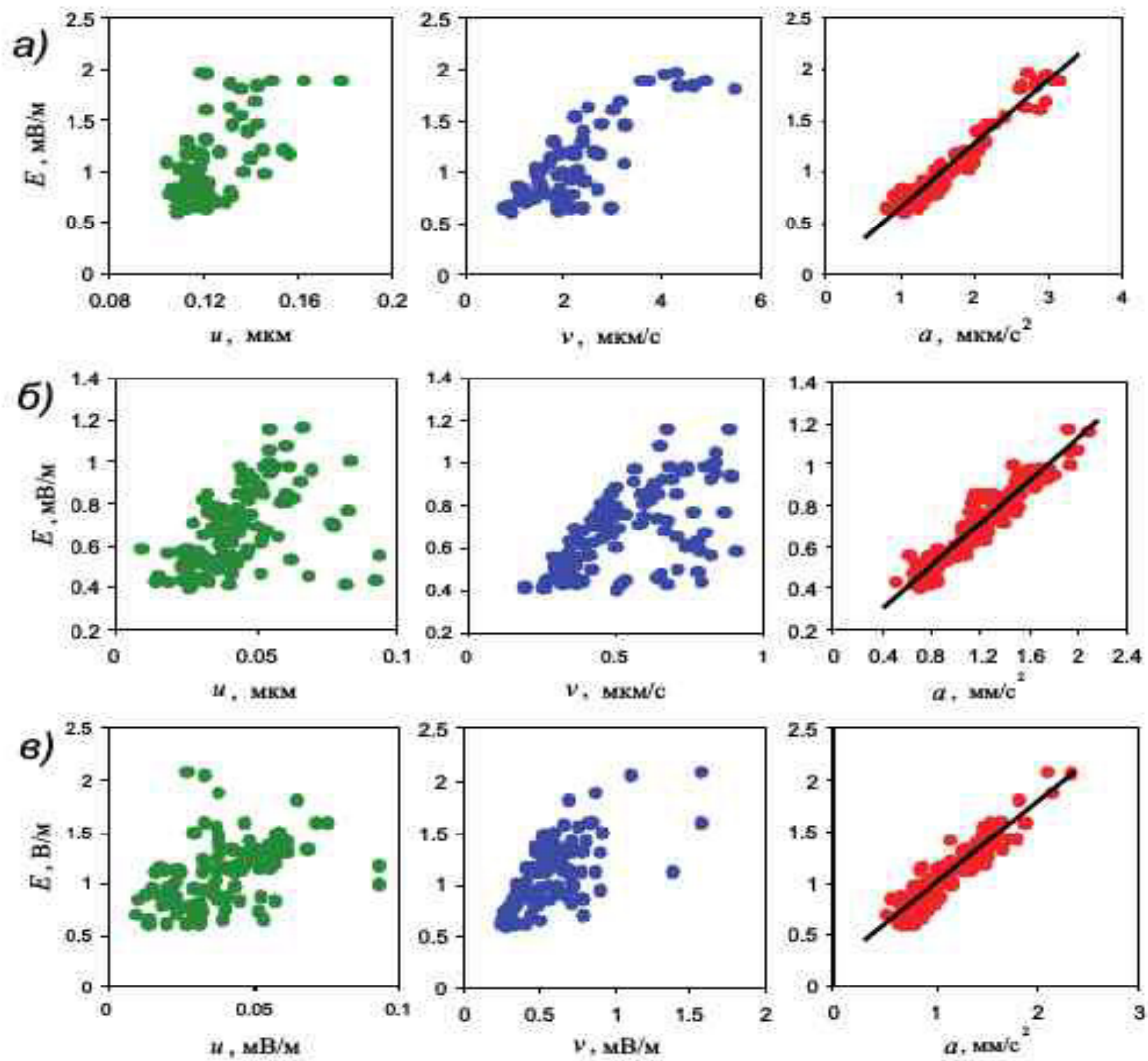

Figure 1: Examples of correlation relations between electric pulse amplitude in the ground $\mathrm{E}$ and the parameters accompanying seismic pulses; the recording in the zone of Tunkinskiy fault for three sessions [7]. 


\section{DEFORMATION-ELECTROMAGNETIC INVESTIGATIONS}

The paper presents the investigation results of the relation of lithosphere deformation activity and natural electromagnetic radiation of EVL-VLF range propagating in the atmosphere.

As the initial data we used the results of recordings of a laser strainmeter and a recording system for electromagnetic ELF-VLF radiation (vertical electric and three mutually orthogonal magnetic components of the field) recorded by the antennas installed at "Karymshina" Geophysical Station of IKIR FEB RAS $\left(52.82^{\circ} N, 158.13^{\circ} \mathrm{E}\right)$. The station is located in the region of intersection of regional faults of the Malko-Petropavlovsk zone of the transverse dislocation of the north-western orientation, in the Paratunska sliding zone at the foot of the extrusive dome of Goryachaya mountain. This territory is characterized by low level of industrial interference and high microseismicity.

The strainmeter has the relative deformation measurement accuracy of not less than $10^{-8}$. The strainmeter sampling frequency is $1 \mathrm{kHz}$.

Sensitivity of the system, recording electromagnetic field, with respect to the magnetic component is not less than $2 \cdot 10^{-7} \mathrm{nT} / \mathrm{Hz}^{1 / 2}$ and $6 \cdot 10^{-8} \mathrm{~V} /\left(\mathrm{m} \mathrm{Hz}{ }^{1 / 2}\right)$ with respect to the electric component.

The recording was carried out in a digitized form with the sampling frequencies of $48 \mathrm{kHz}$ and $44.1 \mathrm{kHz}$.

For the analysis we applied the results of filtration of initial signals in the frequency range of $55 \div 495 \mathrm{~Hz}$. To decrease the industrial noise, we removed a band set of $10 \mathrm{~Hz}$ wide, centered relatively the frequencies, multiple of the industrial frequency ( $50 \mathrm{~Hz}$ ). During the previous investigations we determined that this frequency range contains intensive enough seismotectonic radiation when the effect from radiation of atmosphericlightning origin is low. After the filtration, electromagnetic signal module is averaged in a window of $1 \mathrm{~s}$ wide. In the result, an electromagnetic signal is an average value of field 1second averaged amplitude filtered from industrial noise. The measurements were carried out for the period from 01.02.2018 to 31.03.2018. After the removal of flawed data, 1294 405 second counts were left. It corresponded to the total duration of the change interval of more than 15 days.

Two-dimensional histograms for joint event frequency density logarithm of deformation acceleration and electromagnetic field characteristics. Axis $\mathrm{X}$ is deformation acceleration. Axis Y for (Def - Ez) is the vertical electric field amplitude; (Def - Hwe) is the magnetic field WE component amplitude; (Def-Hz) is the magnetic field $\mathrm{Z}$ component amplitude; (Def-Impedanse) is $E_{z} / H_{w e}$ amplitude; (Def-Impedanse1) is $E_{z} / H_{z}$ amplitude; (Energy) is $E_{z} \cdot H_{w e}$ (strengh). All values are in relative units. 
To analyse the relation between the deformation activity and natural electromagnetic field, we applied the analysis of two-dimensional histograms of deformation acceleration value depending both on direct field characteristics (field amplitude of horizontal and vertical magnetic and vertical electric components) and indirect characteristics (amplitude relations of vertical electric and vertical magnetic, vertical electric and horizontal magnetic and the product of vertical electric on horizontal magnetic giving an idea on field strength). The indirect relative characteristics are mainly determined by rock parameters and the character of the processes occurring in it. All the histrograms are represented in Fig. 2.
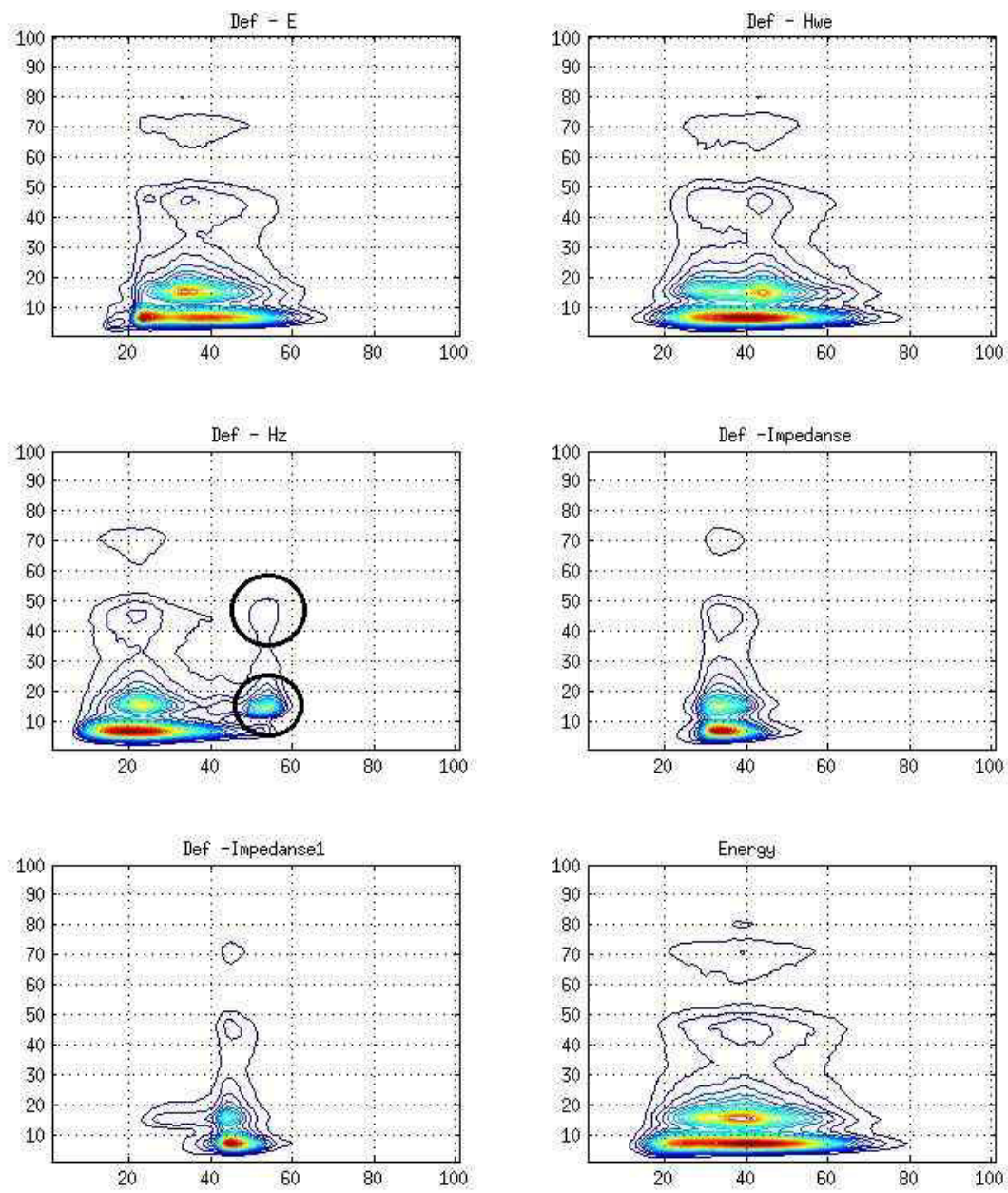

Figure 2: Two-dimensional histograms for joint event frequency density logarithm of deformation acceleration and electromagnetic field characteristics. Axis $\mathrm{X}$ is deformation acceleration. Axis $\mathrm{Y}$ for (Def - Ez) is the vertical electric field amplitude; (Def - Hwe) is the magnetic field WE component amplitude; (Def-Hz) is the magnetic field $\mathrm{Z}$ component amplitude; (Def-Impedanse) is amplitude; (Def-Impedanse1)is amplitude; (Energy) is (strengh). All values are in relative units. 
Several points of attraction, corresponding to the maxima of joint electromagneicdeformation event frequency density, are observed on the historgrams.

First of all, we should pay attention to the highest maximum corresponding to electromagnetic field amplitude small values during quite a wide range of deformation acceleration. It can be treated as the absence of electromagnetic manifestation of the deformation.

The other maxima indicate the presence of several kinds of stationary conditions of process generation and behavior. These conditions are both different values of threshold stress between elastic and plastic deformations for different rocks and their position, and different efficiency of mechanic-electromagnetic transformations determined by rock petrographic composition in the region of deformation processes.

If we assume that event occurrence frequency maxima are determined by elastic-plastic deformation threshold value, we can say that there are two thresholds corresponding to two ranges of deformation acceleration with the values of $15 \div 30,35 \div 50$ conventional units. This may correspond to the rocks of two characteristic petrographic compositions and two different physical conditions (for example, to different temperatures).

In a similar way we can estimate the maxima corresponding to the characteristic values of mechanic-electromagnetic transformations $5 \div 8,13 \div 17,43 \div 48$ and $65 \div 75$ conventional units.

We can assume that these maxima are determined by different values of mechanicelectromagnetic transformations of minerals in a deforming region. The values of maxima number (points of attraction) varies on different histograms.

The largest number of maxima on histogram (Def-Hz) is 7.

We should note the significant differences of histograms for different parameters. There are two maxima on the histogram, comparing acceleration with the vertical magnetic component(Def-Hz). They are marked by circles. Large acceleration values, which are weakly defined on the histogram with magnetic field horizontal direction (Def-Hwe), correspond to these areas. It allows us to assume that the efficiency of mechanicelectromagnetic transformations is an anisotropic value and is associated with gravity direction.

\section{RESULTS}

1. Comparison of crust deformation acceleration with the Earth natural electromagnetic field parameters has been carried out for the first time.

2. Two-dimensional histograms of joint synchronous events of deformation acceleration and natural electromagnetic field parameters showed clearly defined relation for the seismically active region.

3. We've made an assumption on the existence of stationary formation regions for deformation processes and mechanic-electromagnetic transformations corresponding to 
deforming regions with different petrographic composition of rocks and physical conditions of its position.

\section{REFERENCES}

1. M. Hayakawa Electromagnetic phenomena associated with earthquakes, (Radio Sci.Bull. - 2007)

2. V.N. Uvarov Geophysical Journal, 34, 6, 2012,

3. V. Surkov, M. Hayakawa Ultra and Extremely Low Frequency Electromagnetic Fields. (Springer Tokyo Heidelberg New York Dortrecht London, Springer Japan 2014)

4. V.N.Uvarov, Geophysical Journal, 38, 6, (2016)

5. V.N. Uvarov Bulletin of KRAESC. Phys.-Math. Science. 1, 12, (2016)

6. L.D. Landau , E.M. Lifshits Theoretical Physics. Study guide in 10 volums. Field theory, (M: Nauka, 1988)

7. V.V. Adushkin, A.A. SpivakPhysical fields in near-surface Geophysics. (M: GEOS, 2014) 